\title{
La technique et la chair
}

De l'ensarkosis logou à la critique de la société technicienne chez

Bernard Charbonneau, Jacques Ellul et Ivan Illich

Daniel Cérézuelle

\section{(2) OpenEdition}

12 Journals

Édition électronique

URL : http://journals.openedition.org/ress/323

DOI : $10.4000 /$ ress.323

ISSN : 1663-4446

Éditeur

Librairie Droz

Édition imprimée

Date de publication : 1 août 2005

Pagination : $5-30$

ISBN : 2-600-01027-0

ISSN : 0048-8046

\section{Référence électronique}

Daniel Cérézuelle, "La technique et la chair », Revue européenne des sciences sociales [En ligne], XLIII-132 | 2005, mis en ligne le 29 octobre 2009, consulté le 19 avril 2019. URL : http://

journals.openedition.org/ress/323; DOI : 10.4000/ress.323 


\section{LA TECHNIQUE ET LA CHAIR De l'ensarkosis logou à la critique de la société technicienne chez Bernard Charbonneau, Jacques Ellul et Ivan Illich}

Théologie et critique de la civilisation industrielle: Au lendemain de la deuxième guerre mondiale le théologien Karl Barth regrettait que la théologie n'ait pas accordé suffisamment d'importance à la question de l'incarnation et à ses implications morales; il suggérait que cette négligence a contribué à l'indifférence des modernes à l'égard de notre corps «qui nous rattache suffisamment au monde des plantes et des animaux ». Il y voyait aussi l'origine de «la grave dépréciation que l'œuvre humaine a subi» dans la société industrielle ${ }^{1}$. Peut être pensait-il que si l'Occident chrétien avait accordé une plus grande importance à cette dimension de la révélation biblique, la civilisation moderne aurait pu prendre un autre cours. Or il est remarquable que le protestant Jacques Ellul, l'agnostique Bernard Charbonneau et le catholique Ivan Illich, se réfèrent aussi à cette notion d'incarnation comme à un des fondements de leur critique du monde industriel et de la société technicienne ${ }^{2}$. Cependant, pour autant que je sache, aucun d'entre eux n'a pris la peine d'expliciter de manière approfondie ce rapport entre la question de l'incarnation et leur critique sociale ${ }^{3}$. Pour le lecteur qui s'intéresse à la pensée de ces trois auteurs, ces allusions à la notion d'incarnation peuvent paraître bien vagues et déconcertantes. Les remarques qui suivent ont pour objectif de

1 Barth, Karl L'humanité de Dieu, pp. 34 et 35. Traduction française de Jacques de Senarclens, Labor et Fides, Genève 1956, 56 p.

2 Voir les textes suivants:

«A la rigueur nous pouvions entrer dans la perspective d'un personnalisme, dégagé de l'individualisme du XIX ${ }^{e}$ siècle, retrouvant les dimensions essentielles de l'homme, insistant sur l'unité de l'être humain, sur l'incarnation, sur l'engagement en fonction d'une décision personnelle vraiment choisie...» Jacques Ellul, Introduction à la pensée de Bernard Charbonneau in revue Ouvertures, Cahiers du Sud-Ouest, $\mathrm{n}^{\circ}$ 7, 1985, p. 41. Pour éclairer cette référence je me suis reporté à un des premiers écrits théologiques d'Ellul: Présence au monde moderne, dont je citerai quelques passages dans la suite de cette étude. Mais même dans ce livre où Ellul voulait exposer les fondements théologiques de sa critique sociale, le lien entre d'un côté la critique de la technique et de l'Etat et d'un autre côté la notion d'incarnation reste plus affirmé qu'expliqué.

Jean Robert, En mémoire d'Ivan Illich, Cuernavaca décembre 2002. Texte diffusé par le Cercle des lecteurs d'Ivan Illich, Lausanne, 2003.

Ivan Illich, La perte du monde et de la chair, traduit de l'allemand par Jean Robert. In La perte des sens, Fayard, Paris, 2004, 360 p.

Tous ces textes restent allusifs et j'ignore si Karl Barth est allé plus loin qu'eux.

3 Un autre critique de la civilisation industrielle l'a fait de manière plus explicite, c'est Hans Jonas. De l'étude de la gnose à son éthique environnementaliste, en passant par sa philosophie de l'organisme, la question de l'incarnation a toujours été un des fils conducteurs de sa pensée. 
préciser ce rapport et de mieux comprendre pourquoi ces trois auteurs se sont adossés à cette notion d'incarnation pour critiquer la conception moderne de la liberté et le rapport au monde naturel qui en découle dans la civilisation industrielle. Pour cela il m'a semblé important de caractériser la vision du monde et la compréhension de la liberté qui découlent du caractère central de cette notion d'incarnation dans la théologie chrétienne traditionnelle; puis j'ai cherché à dégager le lien entre cette vision du monde et la critique de la civilisation industrielle chez chacun de ces trois auteurs. Enfin je propose quelques réflexions plus philosophiques sur l'importance de la notion de chair pour réfléchir aux limites que l'on pourrait assigner aux technosciences.

Bien entendu je n'ai pas la prétention de définir ce que devrait être une pensée chrétienne orthodoxe. Il est clair, et l'histoire en témoigne suffisamment, que selon les sensibilités religieuses l'incarnation du Christ peut être interprétée de diverses manières. Il n'est que trop évident que du Christianisme sont issus des courants spirituels qui entretiennent la même méfiance à l'égard du corps et de la sensibilité ou bien la même indifférence à l'égard de la nature que certaines religions non chrétiennes. On peut même penser que ces courants sont ceux qui ont eu une grande influence dans l'histoire de l'Occident. Cependant, d'un même réservoir peuvent s'écouler des filets d'eau qui prendront des directions différentes pour irriguer et fertiliser des terroirs différents. Il s'agit ici seulement de suivre un de ces filets d'eau pour comprendre comment et pourquoi au vingtième siècle certains esprits ont cru pouvoir se référer à une certaine vision judéo-chrétienne de l'homme comme chair pour exprimer, voire pour légitimer leur révolte contre la civilisation industrielle. Ce faisant j'ai pris le risque de m'aventurer dans un domaine qui n'est pas le mien puisque je ne suis ni théologien ni croyant. S'il m'a semblé utile de m'engager sur ce terrain c'est pour deux motifs. D'abord pour mieux comprendre ce que ces trois auteurs, Charbonneau, Ellul et Illich, ont en commun. Ensuite, considérant que ces trois penseurs ont mis le doigt sur de vrais problèmes, j'espère ainsi mieux identifier certains des enjeux fondamentaux de la critique de la modernité industrielle. Cependant le lecteur ne doit pas oublier que faute de pouvoir m'appuyer sur des textes explicites, je ne peux proposer ici qu'une reconstruction qui a un caractère largement hypothétique. Et si, faute de compétences, ces quelques pages ne sont pas à la hauteur de mes objectifs, ce n'est pas bien grave: on peut toujours espérer que leur lecture donnera envie à quelqu'un de plus compétent que moi de reprendre la tâche et de la mener à son terme.

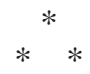

\section{DEUX MODÈLES DE LA PERFECTION}

Libre comme l'air: Dans beaucoup de religions la perfection s'atteint par un mouvement de désincarnation: habité par une puissance surnaturelle, le chamane peut quitter son corps et évoluer dans le monde des esprits; dans toutes les religions des saints peuvent voler, devenir immortels etc. Les mystiques veulent toutes affranchir le sujet de sa condition d'être vivant dans un corps soumis à des limites spatio-temporelles, que ce soit par l'extase, par la vision en Dieu, la contemplation du tout etc. Cette auto-déification par la désincarnation de l'esprit 
est aussi le but de bien des philosophies spéculatives qui invitent l'homme à libérer son esprit des contraintes, des imperfections et des limites de la pensée humaine grâce au pouvoir du concept. Ce désir de dépasser l'humain, de faire advenir le post-humain ou le trans-humain, c'est aussi un des moteurs de l'aventure technicienne ${ }^{4}$. Ces divers modèles de la perfection humaine ont à leur source une même expérience, fort commune, de l'absence de liberté.

Constamment nous avons l'impression que notre volonté se heurte à la résistance que lui oppose la réalité. Non seulement nous sommes contraints de vivre dans un monde social peuplé de personnes dont les projets font obstacles aux nôtres, mais encore nous vivons dans un monde naturel dont l'inertie et le poids résistent à nos projets. Que ce soit hors de nous ou jusques en nous-mêmes dans l'expérience de l'effort et de la peine qui résultent de nos limites physiques, le monde du corps nous apparaît souvent comme cause d'imperfection et finalement de mort. L'expérience de la limite et de l'obstacle est donc toujours vécue comme un amoindrissement de notre liberté, comme une imperfection, et nous nous imaginons volontiers qu'être libre c'est abolir ce qui résiste à notre volonté et ce qui limite notre puissance. Au contraire je m'éprouve comme libre quand rien ne me résiste, quand je suis sans liens, qu'ils soient naturels ou sociaux. C'est pourquoi nous nous représentons spontanément la liberté totale sur le modèle la toutepuissance qui fait partie des attributs de la perfection divine. Il n'est donc pas étonnant que pour bien des esprits la perfection consiste à être libéré de la nature, et se libérer consiste donc à défaire les liens qui lient l'esprit aux lois de la nature corporelle. Accéder à la liberté c'est acquérir la puissance mentale, intellectuelle ou physique de libérer l'esprit des diverses limites qui résultent du caractère naturel de l'existence et en particulier du lien qui l'attache au corps. Tout ce qui libère l'esprit des limites liées à l'incarnation de l'existence est donc vécu comme facteur de perfection. D'où la constance des symbolismes ascensionnels et de la valorisation de la transparence et de la verticalité dans les représentations de la perfection humaine. Cette représentation de la perfection peut susciter une grande méfiance à l'égard de la vie des sens qui nous enchaînent au monde. A l'opposé des représentations sacro-magiques d'un univers vivant, habité par des puissances avec lesquelles l'homme doit sans cesse composer, la valorisation d'une transcendance désincarnée peut également favoriser une profonde indifférence de la pensée morale à l'égard des dimensions sensibles de la vie quotidienne qui sont considérées comme contingentes. Un tel état d'esprit est peu favorable à une révolte devant le saccage de la nature et la dépersonnalisation de la vie quotidienne; au contraire il peut tout à fait s'accommoder avec la fascination pour la puissance technique et conduire à saluer dans tout progrès de la puissance instrumentale le moyen d'affranchir l'esprit des servitudes d'une corporéité vécue comme obstacle.

Sur la terre comme au ciel: La religion juive puis la révélation chrétienne rompent avec cette aspiration à la perfection désincarnée. A une humanité obsédée par le désir d'échapper à sa condition («vous serez comme des dieux...»),

${ }^{4}$ Voir les ouvrages de Jean Brun, en particulier La machine et le rêve, Paris, La Table ronde, 1992. 
le Dieu de la Bible va donner aux hommes l'exemple d'une perfection inouïe - et scandaleuse - en s'incarnant dans ce monde, sans perdre de sa perfection. En effet de la naissance de Jésus la bible dit «et le verbe se fit chair». Cette ensarkosis logou peut être interprétée de diverses manières. Par exemple une théologie du rachat présente couramment cette incarnation comme un sacrifice: afin de délivrer par ses souffrances les hommes du mal et des conséquences de leurs péchés Dieu consent à un amoindrissement et se fait homme sur terre pour y souffrir. Mais on peut comprendre autrement cette incarnation: au lieu d'y voir un amoindrissement on peut voir dans ce mouvement d'incarnation l'expression d'une perfection suprême. En se faisant chair sentante et pensante, existence individuelle active dans le temps et dans l'espace, le verbe donne aux hommes le modèle d'une perfection dans ce monde. Jusque là les hommes pouvaient penser que la perfection qui réalise toutes les aspirations de l'esprit ne peut exister que dans un au delà du monde naturel. Jésus, en assumant la condition d'homme, donne aux hommes l'exemple de la pleine réalisation du spirituel dans ce monde. Comme au moment de la création, le verbe n'est plus cantonné dans l'autre monde. L'idéal peut s'inscrire dans le réel, dans le temps et dans l'espace, dans la vie quotidienne; en la personne de Jésus les hommes en ont l'exemple.

Désormais l'aspiration humaine à la perfection peut s'inscrire dans un espace spirituel nouveau. A la verticalité, la dimension du Père «qui est aux cieux », vient s'associer l'horizontalité, sanctifiée par le Fils. C'est pourquoi à ceux qui vivent dans ce monde il est dit: "Soyez parfait comme votre père dans les cieux est parfait». Sur cette terre il est possible de mener une vie sainte en mettant en pratique la loi d'amour. Il faut pour cela suivre l'exemple de Jésus qui a assumé totalement la condition humaine, qu'il s'agisse de la participation à des banquets bien arrosés ou de l'épreuve de l'agonie.

Etre le corps du Christ: Il est remarquable qu'au terme du récit de la passion, l'Evangile dit que le tombeau où avait été déposé le corps du Christ est trouvé vide. La disparition de ce corps a plusieurs conséquences.

Premièrement: elle atteste que Jésus est toujours vivant et qu'il siège désormais près du Père. Alors que dans beaucoup de religions le parfait abandonne à ce monde naturel sa «dépouille mortelle», comme si le corps était un obstacle à sa totale perfection, une chose inessentielle que l'on peut abandonner à ce monde, Jésus ressuscite avec son corps. On ne peut mieux sanctifier le corps!

Deuxièmement les hommes qui vivent sur Terre ne disposent plus d'un corps à momifier et adorer en un lieu spécial, comme celui de Pharaon ou de Lénine, pour entrer en relation avec la transcendance. Il leur est expressément enjoint de «ne plus chercher sur terre celui qui est aux cieux». Il leur est dit plutôt que «là où deux ou trois seront réunis en mon nom, mon esprit sera avec vous ». Jésus n'a plus de corps sur Terre. Les hommes n'ont plus pour orienter leur désir de sainteté que l'inspiration de l'Esprit de Dieu.

Troisièmement, et par conséquent, il est conféré aux croyants une responsabilité nouvelle, puisqu'il leur est dit: «Vous êtes le corps du Christ». Sans prétendre épuiser la richesse symbolique de cette formule, on peut comprendre cette affirmation comme une injonction faite aux hommes de donner corps dans ce monde à l'esprit du Christ. C'est à chacun des hommes et à eux tous ensemble de faire en sorte que par leurs actes l'esprit de justice d'amour et de liberté trouve la force de s'ins- 
crire dans le monde et de le changer. Faire que tous les jours le verbe se fasse chair, donner corps et réalité dans ce monde, aux exigences de l'esprit, là est la sainteté, là est une nouvelle expérience de la perfection qui doit guider la liberté humaine.

Cette situation réoriente la vie religieuse des hommes non plus seulement vers le haut pour s'y évader mais vers la terre pour y réaliser les exigences de l'esprit en leur donnant un corps. Désormais la perfection ne va plus consister dans l'ascèse qui permet de se désincarner pour échapper au monde ainsi qu'aux puissances de la nature, du corps et de la société qui font obstacle aux exigences de l'esprit; elle consiste à incarner l'esprit d'amour et de liberté; incarner, c'est-àdire le rendre actif, visible et réellement fort dans un monde naturel et social qui, laissé à lui-même, ne connaît que la puissance.

Les deux axes de la croix qui rappellent la mort et la résurrection du Christ rappellent aussi et par conséquent à chaque individu que - comme le Christ - il doit désormais vivre sa liberté à la croisée de deux exigences. D'un côté une exigence de verticalité, d'être en relation avec une vérité spirituelle qui n'est pas inscrite dans la nature («mon royaume n'est pas de ce monde») et d'un autre coté une exigence d'horizontalité et de mise en pratique de la vérité dans ce monde, principalement à travers les rapports que nous entretenons avec notre prochain: là est le Royaume, là est le sens. Donner un corps aux exigences de l'esprit, voici la perfection. Cela, seul un homme, un esprit singulier vivant dans son corps individuel, peut l'accomplir. Et chaque fois qu'il le fait dans l'instant, il le fait aussi pour l'éternités.

Ainsi l'accent mis par la Bible sur l'incarnation oriente donc la liberté de l'homme dans une nouvelle direction. Nous ne sommes plus invités à dépasser la condition de l'homme mais à la vivre totalement. Nous avons vu que dans la plupart des conceptions non chrétiennes de la perfection, l'expérience de la transcendance de l'esprit nourrissait une recherche de diverses formes de déliaison visant à annuler les liens qui font obstacle aux aspirations de l'esprit.. C'est dans cette déliaison que consiste la liberté, et tout ce qui abolit ces obstacles et contribue à la désincarnation de l'homme est vécu comme facteur de libération. Or, dans une perspective qui, inspirée par l'exemple du Christ, reconnaît l'incarnation comme une dimension centrale de l'existence humaine, la sainteté n'est plus dans la déliaison mais plutôt dans l'acte d'incarnation de l'esprit et de ses valeurs. Voilà à quoi est appelée la liberté humaine. Et comme cette exigence d'incarnation ne connaît pas de limites, ce n'est plus seulement au cours de moments spéciaux de leur vie spirituelle que les hommes sont appelés à réaliser cette incarnation: désormais investis de la liberté des enfants de Dieu, c'est dans toutes les dimensions de leur vie, y compris de leur vie quotidienne qu'ils doivent agir pour donner un contenu concret à leurs valeurs. C'est donc à l'aune de l'expérience de la totalité de la vie quotidienne, telle que chaque individu peut en faire l'expérience, qu'il convient de juger la valeur des entreprises humaines : c' est à ses fruits que l'on reconnaît l'arbre.

$$
\text { * * }
$$

5 «Celui qui a compris ces paroles et les met en pratique, celui-là a bâti sa maison sur le roc» Voir le journal personnaliste Hic et nunc, brièvement publié dans les années trente par un des fondateurs historiques du mouvement écologiste: Denis de Rougemont, auteur de Penser avec les mains. 


\section{TROIS CRITIQUES \\ DE LA DÉPERSONNALISATION TECHNICIENNE}

Liberté et incarnation chez Bernard Charbonneau : Toute la pensée de Charbonneau découle d'une conviction fondamentale, à savoir que la civilisation industrielle ne peut pas répondre de manière satisfaisante à deux besoins de l'homme: le besoin de nature et le besoin d'une action personnelle, autrement dit: de liberté. C'est pourquoi toute son œuvre est une invite à chercher un autre modèle de civilisation qui fasse sa place au besoin de nature et de liberté de l'homme. Or, c'est bien parce qu'il pense que l'incarnation est une dimension centrale de la condition de l'homme qu'il pense aussi que l'impossibilité de satisfaire ces besoins a pour effet la dépersonnalisation de l'existence. Comme il l'écrit dans Le système et le chaos, le «développement incontrôlé menace l'homme dont l'esprit s'incarne en un corps $»^{6}$.

Agnostique, Charbonneau se reconnaissait volontiers comme «Post-chrétien». Par là il se définissait comme héritier non pas d'une conception mais d'une expérience de la liberté personnelle, dont il pensait qu'elle a été transmise à l'Occident par la tradition juive et la révélation chrétienne. Il savait aussi que sa pensée s'inscrit dans une longue tradition augustinienne de méditation sur l'existence. Fidèle à cette tradition, il laisse aux philosophes patentés l'élucidation des conditions transcendantales ou métaphysiques de la liberté: expliquer comment elle est conceptuellement possible dans un univers physique soumis au déterminisme, cela ne l'intéresse pas. Ce qui l'intéresse c'est de comprendre comment elle peut être vécue et, comme Kierkegaard, il est convaincu qu'il n'y a pas de système de l'existence: «la réalité de la liberté n'est pas dans les preuves de la science et de la philosophie - elles te l'assureraient que tu l'aurais déjà perdue - mais dans la personne vivante.» ${ }^{7} \mathrm{C}^{\prime}$ est pourquoi, alors que le souci d'incarnation sous-tend toute l'œuvre de Charbonneau, ce dernier ne s'est pas préoccupé d'élucider conceptuellement cette notion. Ce n'est que vers la fin de sa vie qu'il est devenu plus explicite. Deux textes inédits apportent un éclairage précieux sur la centralité des notions d'incarnation et d'individualité, qui d'ailleurs sont pour Charbonneau inséparables: Le premier est une étude d'une trentaine de pages intitulée «Nicolas Berdiaef: le chrétien, individu ou personne ? ${ }^{8}$ Il s'agit d'un des rares textes dans lesquels Charbonneau propose une explicitation «philosophique» de sa conception de la personne et de la réalisation de la liberté dans l'individu. Le deuxième texte, de trois pages seulement, intitulé «Incarner», atteste bien de la centralité des notions d'incarnation et de chair dans la pensée de Charbonneau. Il conclut le bref opuscule Trois pas vers la liberté ( 9 p.) rédigé par Charbonneau peu de temps avant sa mort en guise de postface à l'ensemble de son œuvre.

Pour cet auteur l'incarnation est donc une dimension centrale de l'existence. Etre libre c'est, précisément, accepter et non fuir la tension entre un impératif

- Charbonneau, Bernard, Le système et le chaos, p. 128, Economica, Paris, 1990, 290 p.

Je Fus p. 140, Opales, Bordeaux, 2000, 235 p.

8 Ce texte constitue le troisième chapitre de l'opuscule inédit Quatre témoins de la liberté: Rousseau, Montaigne, Berdiaef, Dostoïevski (104 p.). 
spirituel et les difficultés à l'incarner dans la nature et dans la société. Or cela seul un individu peut le réaliser dans sa vie: «entre le réel et la terre, entre l'idéal et le réel, il faut un médiateur et il n'y en pas d'autre qu'un homme; pour s'incarner l'esprit n'a jamais usé d'autre biais.» ${ }^{9} \mathrm{Il}$ en résulte que pour l'homme le rêve d'une liberté totale est littéralement insensé car la liberté ne peut être un état; elle consiste en un effort de libération qui aboutit plus ou moins ${ }^{10}$.

C'est pourquoi Charbonneau ne cesse d'affirmer qu'une pensée qui n'est pas mise en pratique dans la vie quotidienne est dérisoire et par conséquent que rien de ce que vit l'individu n'est insignifiant puisque chaque circonstance de la vie quotidienne est l'occasion pour chacun d'entre nous de mettre en pratique nos valeurs.

Par ailleurs si Charbonneau est convaincu qu'il est vital pour la pensée de se traduire par des actes qui lui donnent une réalité matérielle, il est également convaincu que parce que l'homme est un être de chair, les conditions matérielles dans lesquelles il vit sont de la plus haute importance spirituelle. Comme il est attentif à la globalité de la personne il se refuse à privilégier certaines conditions matérielles au détriment des autres. Par exemple pour juger l'appareil productif d'une société il faut tenir compte non seulement du niveau de consommation mais aussi des conditions qui sont faites à la sensibilité dans la vie quotidienne.

C'est ce sens de l'incarnation qui en politique a conduit Charbonneau à répudier les théories libérales ou socialistes, qui réduisent l'expérience de la liberté à son concept et à des conditions isolables, coupées de leur fondement, c'est-à-dire de l'expérience vivante du sujet. C'est aussi ce qu'il reproche à la fascination moderne pour l'efficacité économique ou technique. De telles démarches sont fondées sur une représentation abstraite de la vie et, au nom d'une conception désincarnée de la liberté, s'accommodent trop facilement de la dépersonnalisation de la vie par la science, la technique, l'Etat, l'économie. Ce sont ces diverses formes de dépersonnalisation de l'existence que Charbonneau décrit et analyse dans ses livres. Il montre comment la croissance des moyens et le progrès de l'efficacité, qui jusqu'à un certain point favorisent la liberté, ont pour contrepartie une autonomisation des structures qui favorise la montée, dans tous les domaines, des dégâts du progrès. Dégâts écologiques, politiques et sociaux, mais également spirituels. En effet, qu'il s'agisse du progrès de l'organisation institutionnelle ou de celui de la puissance technique et industrielle, il arrive un moment où la croissance des appareils prive chaque individu de la possibilité d'incarner ses valeurs dans ses actions concrètes. La guerre totale, l'arme absolue, le totalitarisme aussi bien que la dévastation écologique de la planète sont le résultat de «la terrible logique de la science et de l'Etat abandonnées à eux-mêmes $»^{11}$. Evoquant le danger nucléaire il écrit: «La fin de la terre des hommes serait la conclusion d'une désincarnation progressive; la passion de connaître pour connaître et celle de dominer pour dominer se seraient conjuguées avec le recul progressif de l'esprit devant le monde. La force fuyant l'esprit, l'esprit fuyant la force, plus vertigineu-

\footnotetext{
${ }^{9}$ Charbonneau, Bernard, Je Fus, p. 12.

${ }^{10}$ Charbonneau, Bernard, Le sens, texte inédit.

${ }^{11}$ Charbonneau, Bernard, L'Etat p. 421, Economica, Paris, 1987, 449 p.
} 
sement que peuvent se fuir les nébuleuses.» ${ }^{12}$ Méditant sur la montée en puissance des appareils et en particulier de l'Etat, qui caractérise le monde moderne, il constate que «De ma pensée à cette réalité la distance est telle que je me condamne à une pensée désincarnée et une pensée sur l'Etat ne peut être mue que par un tout puissant impératif d'incarnation $»^{13}$.

Pour résister à cette dépersonnalisation Charbonneau estime qu'une critique purement intellectuelle ou scientifique ne suffit pas. Car même si elle suscite une action, cette action sera inspirée par des raisons partielles et sera donc dangereusement unilatérale. La critique et la résistance ne sont possibles que si elles sont nourries par l'amour de la vie et l'attention à toutes les formes de bonheur que la vie sensible nous apporte. Dès 1939 , réfléchissant à ce que devrait être une révolution personnaliste, il écrivait «La révolution vise la délivrance de l'homme de chair et d'esprit, c'est-à-dire que les révolutionnaires doivent penser beaucoup plus près que l'économiste ou le politicien: à la vie que nous menons et à nos prochains $»^{14}$. Tel est le terreau existentiel dans lequel s'enracine l'appel de Charbonneau à associer nos libertés pour maîtriser la «Grande Mue» qui, selon lui, résulte de la montée en puissance de la science et de la technique.

C'est ainsi, par exemple, que la critique charbonnienne de l'industrialisation de l'agriculture découle de cette conviction qu'il ne saurait y avoir de liberté qu'incarnée, que c'est en s'incarnant que la liberté est portée à son plus haut point et que c'est dans les taches apparemment les plus humbles que doivent prendre corps les aspirations de l'esprit. C'est pourquoi la forme et les modalités de la relation que l'homme entretient avec la terre constituent un enjeu humain essentiel et ne doivent pas être abandonnée aux seules lois de l'efficacité technique et de la rentabilité. Au contraire: il faut juger l'innovation agricole non seulement en fonction des gains de productivité mais aussi en fonction des diverses conséquences qui en résultent pour la totalité de la personne et il n'hésite pas à aborder le problème agricole du point de vue de la vie quotidienne sensible. S'il défend un mode paysan (ce qui ne veut pas nécessairement dire traditionnel) d'agriculture ce n'est pas seulement pour préserver la biodiversité ou les grands équilibre écologiques de la planète, c'est d'abord parce qu'il éprouve un attachement charnel à la beauté, à l'harmonie et à la diversité des paysages quelle produit. La beauté des campagnes donne l'exemple d'un accord, d'un compromis réussi et partout différent entre les exigences de l'esprit humain et les contraintes naturelles. A l'opposé, la diffusion de l'agriculture industrielle a pour effet la banalisation et l'uniformisation des paysages et des sociétés locales, l'affadissement des nourritures, la monotonie du travail: autant de formes d'appauvrissement de la vie sensible qui résultent d'une exploitation destructrice des ressources naturelles et qui lui paraissent le contraire d'un véritable progrès.

L'humiliation de la chair chez Ivan Illich: Illich est très connu pour sa critique acerbe des professions et des institutions: de l'Ecole, de la Santé et des transports. Ces critiques particulières sont autant de volets d'une critique plus

\footnotetext{
${ }^{12}$ Op. cit. p. 422.

${ }_{13}$ Op. cit. p. 10.

${ }^{14}$ Bernard Charbonneau, «Réformisme et action révolutionnaire», revue Esprit n 77, 1939.
} 
globale de ce que les américains ont dénommé le technological fix, c'est-à-dire la mise en place de procédures techniques chargées de répondre par des procédures professionnalisées au problèmes posés en premier lieu par le progrès technique. Ivan Illich fait deux reproches complémentaires à la civilisation industrielle et technicienne: premièrement la prolifération des spécialistes et des professionnels prive les personnes et les groupes de la capacité de maîtriser leur vie quotidienne; deuxièmement elle ôte aux hommes la capacité à orienter leur action en fonction de leur expérience du monde, expérience qui est d'abord sensible et charnelle.

Le raison technique, comme la raison économique et l'institution rationnelle ne peut se déployer qu'en ignorant l'unité charnelle de la vie et l'importance du symbolique. La contreproductivité et l'hétéronomie produite par les techniques et les institutions professionnalisées, jouissant au nom de leur technicité de ce qu'Illich appelle un monopole radical, sont les effets - ou les symptômes - de ce décalage entre le mode d'être au monde humain, et les représentations conceptuelles et rationalisées, auxquelles les modernes ont recours pour expliquer et organiser leur vie. Prolongeant les intuitions de la phénoménologie, Illich s'attache à montrer que le type de rationalité qui se répand avec la civilisation technicienne et industrielle désincarne le monde vécu pour mieux opérationnaliser le réel.

Le déploiement du monde de la technique requiert la dévalorisation du corps et de l'expérience sensible. Or celle-ci fonde des savoirs qui sont souvent plus riches, et complexes et surtout plus appropriables que les savoirs «scientifiques ou techniques », ou provisoirement certifiés comme tels, savoirs qui sont fondés sur une expérience soi-disant objective mais nécessairement toujours partielle, fondée en dernier recours sur la mise à l'écart de certaines dimensions du monde vécu. De tels savoir sont forcément spécialisés et incompréhensibles par le non spécialiste. D'où deux types de problèmes qui ne peuvent que s'aggraver avec la civilisation technicienne: D'une part la contre-productivité fréquente de ces savoirs et de ces techniques lorsque leur puissance et leur diffusion dépassent un certain seuil. Au-delà de ce seuil leur partialité, qui rend possible leur efficacité, a des effets inévitablement destructeurs. D'autre part Illich s'est longuement attaché à montrer la perte d'autonomie ainsi que la diffusion d'une culture de la dépendance qui résultent du progrès de ces techniques. On voit que pour Illich la question du corps propre et celle de l'appropriation des savoirs et des pouvoirs sont solidaires. C'est pourquoi il nous invite à évaluer les techniques en fonction de deux critères: quelles sont leurs conséquences sur l'autonomie des individus et des groupes, et quelles sont leurs conséquences sur la vie du corps?

Pour répondre à cette seconde question, influencé semble t-il par la philosophie néo-thomiste de Jacques Maritain, Illich semble parfois inscrire sa pensée dans l'optique d'une conception essentialiste de la nature humaine et dénoncer l'altération des fonctions du corps comme une sorte de péché contre l'ordre de la création, ce qui se traduit par l'apparition dans certains textes de qualificatifs tels qu'innommable, diabolique, monstrueux etc. Mais il n'en reste pas là et dans d'autres passages sa critique de l'intempérance technicienne et de la dépersonnalisation de la vie qui en découle se fonde sur une approche plus existentielle et phénoménologique des effets de la technique moderne. Plusieurs de ses textes s'attachent à montrer comment certaines innovations techniques et le rapport au monde qu'elles instaurent, peuvent d'un même mouvement nous priver de l'usage 
de notre corps et d'un rapport charnel au monde, des connaissances appropriables qui en découlent et enfin d'une prise personnelle sur notre existence.

Ivan Illich était prêtre et c'est volontairement qu'il n'a pas exprimé publiquement les fondements théologiques de sa critique sociale car, dit-il, «dans la tradition la plus récente de l'Eglise Catholique Romaine, celui qui prétend parler comme théologien se revêt de l'autorité que lui confère la hiérarchie. Je ne prétends pas être investi de ce mandat ${ }^{15}$. Cependant, comme le montrent Jean Robert et Valentine Borremans ${ }^{16}$ la critique illichienne de la technique s'enracine bien dans une pensée de l'incarnation. Barbara Duden ${ }^{17}$ confirme elle aussi cette interprétation de la pensée Illichienne. Elle montre que ce thème de la chair soustend l'œuvre écrite d'Illich et que, par exemple, pour ce dernier il serait impossible de comprendre l'avènement de la conception moderne de la santé, et même la conception post-moderne du moi, sans une mise en perspective historique de la notion de chair et de sa décomposition culturelle. Mais elle souligne également le fait qu'Illich traite de la chair de manière «apophatique », c'est-à-dire en creux, de la même manière que l'on parle de Dieu dans la tradition de la théologie négative, en disant non ce qu'il est (et qui échappe au pouvoir de nos concepts) mais ce qu'il n'est pas. Selon Barbara Duden cette réserve s'explique par le fait que pour «pour lui la chair nous oriente inexorablement vers l'Incarnation, vers le mystère qui est dans le monde de sa foi, et en fin de compte vers la Croix ». Au fond, ce qu'Illich reproche au technicisme occidental c'est d'avoir trahi le mystère de l'incarnation $^{18}$ et de la nécessaire proportionalité - on pourrait dire aussi de l'union, au sens conjugal, ou encore du bon accord - entre le verbe et la chair, proportionnalité qui, selon lui, doit orienter la vie humaine et dans toutes ses dimensions. Dans cette perspective il est important pour Illich que le corps propre et son expérience sensible du réel soit le principal médiateur de notre rapport à la réalité. L'homme est chair et c'est en tant que chair que nous le rencontrons. Or précisément la technicisation de l'existence a pour contrepartie la rupture de cette union entre l'esprit (le verbe) et la chair, union qui selon lui est pourtant constitutive de notre humanité. Illich voit même dans la vocation techniciste de l'Occident le fruit du rejet de ce moi de chair qu'il a hérité de la Bible. La modernité progresse en procédant à une désincarnation croissante de l'existence et c'est pour cela qu'il en résulte une dépersonnalisation croissante de la vie et une perte croissante de maîtrise sur notre vie quotidienne, et donc de liberté.

${ }^{15}$ Cité par Borremans, Valentine et Robert, Jean, préface aux Euvres Complètes d'Ivan Illich. Arthème Fayard, vol 1, 792 p., Paris, 2004. Les présentes remarques sur la pensée d'Illich doivent beaucoup à ce texte très pénétrant ainsi qu'à l'étude de Barbara Duden citée ci-après.

16 Ibid.

${ }^{17}$ Duden, Barbara, «The quest for past somatics», In Lee Hoinacki and Carl Mitcham (sous la direction de) The challenges of Ivan Illich, State University of New York Press, 2002.

${ }^{18}$ Charbonneau formule le même reproche dans des termes très proches. Il parle lui aussi de trahison. De même on trouve chez ces deux auteurs l'idée que seule la conscience aigue de la liberté (héritée du Christianisme) a pu conduire à une entreprise qui débouche sur la négation radicale de la liberté. Ce qu'Illich exprime en utilisant la formule théologique selon laquelle Corruptio optimi quae est pessima, Charbonneau l'exprime aussi par la formule La liberté seule peut justifier sa négation. (Je fus p. 195). 
Par exemple, à travers une phénoménologie de la technique de la lecture silencieuse qui s'est développée en occident à partir du douzième siècle, Illich diagnostique que c'est bien la trahison de l'incarnation qui a ouvert la voie à deux possibilités symétriques de corruption: D'un côté une désincarnation sans précédent de la parole et donc de la pensée, libérée de son ancrage charnel; d'un autre côté - et réciproquement - une «incarnation perverse» qui conduit à vouloir donner un statut de réalité concrète et autonome à des concepts chargés ensuite d'organiser notre relation au réel. Dans plusieurs textes Illich s'attache à montrer que cette «incarnation perverse d'entités sans chair» caractérise le monde moderne. Comme le soulignent Valentine Borremans et Jean Robert, commençant avec l'age de la vitesse, elle s'accélère en la multiplication de fausses concrétudes et de pseudo-percepts et culmine en une transformation technogène, c'est-à-dire assistée par la Technique, du sens de la matière. La trahison de l'incarnation prend donc deux formes observables: une lente désincarnation historique de la pensée et, plus récemment, une pseudo-incarnation technogène d'entités intrinsèquement dépourvues de chair que nous utilisons pour penser le corps ou bien la société, et pour agir sur eux. Cette inversion des rapports entre le verbe et la chair favorise forcément une instrumentalisation du corps et plus largement de tout donné naturel et social qui doit être subordonné à des modèles abstraits de connaissance et d'opérativité technique. Ainsi Illich se préoccupe de la désincarnation de la perception de soi qui résulte de la médicalisation des arrangements sociaux et des normes culturelles $^{19}$. Plus généralement encore, c'est bien l'expérience de la désincarnation qui lui semble caractériser le mieux le passage du monde préindustriel à la modernité tecnicienne ${ }^{20}$. C'est dans ce fond spirituel que s'enracinent les maux qui caractérisent selon Illich le monde moderne et qu'il subsume par l'expression humiliation de la chair: la dépersonnalisation de l'existence, la disqualification des savoirs vernaculaires appropriables, la gestion technocratique de la vie par des professionnels spécialisés, la perte de maîtrise sur la vie quotidienne ${ }^{21}$. Alors que l'idéologie progressiste et scientiste est fascinée par le développement de la puissance collective que la science et la technique donnent à l'humanité sur tout ce qui est corporel, ce même soucis de l'incarnation conduit Illich a insister sur la nécessité d'une maîtrise personnelle de nos outils, qu'ils soient techniques, institutionnels ou intellectuels. Pour que l'outil soit digne de ce nom, il faut que ses fins et son utilisation conservent un certain caractère personnel. Comme Charbonneau, et pour les mêmes raisons, Illich considère qu'un des critères de l'évaluation des techniques c'est de savoir si elles augmentent la maîtrise responsable et donc la liberté des personnes, des individus concrets. Or, selon Illich, à l'Age des Systèmes on assiste au démantèlement des fins personnelles. A partir de 1980 les écrits d'Illich peuvent être interprétés comme l'exploration des effets historiques, démontrables et datables, de ces deux versants d'une trahison spécifiquement chrétienne et occidentale de l'exigence d'incarnation, du mystère de la chair, de l'union de l'esprit et du corps qui est au centre du christianisme.

19 Illich Ivan, «la société amortelle» in La perte des sens, 277 p.

${ }^{20}$ Illich Ivan, «la perte du monde et de la chair» in La perte des sens, 355 p.

${ }^{21}$ Déjà dans les années trente le personnaliste Denis de Rougemont, ami de Charbonneau et d'Ellul, critiquait cette désincarnation de la pensée dans son livre Penser avec les mains (1936). 
L'incarnation chez Jacques Ellul: Dans Présence au monde moderne ${ }^{22}$, Ellul expose les grandes lignes de sa conception d'une éthique chrétienne pour un monde moderne dominé par la puissance de la technique et de l'Etat. Il s'agit d'un de ses rares ouvrages qui fait explicitement le lien entre d'un côté sa pensée religieuse et d'un autre côté sa pensée sociale et sa critique de la technique moderne, alors que dans la plupart de ses livres il s'est volontairement appliqué à dissocier ces deux volets de son œuvre. Dès le début de cet ouvrage Ellul inscrit sa réflexion dans l'horizon de l'incarnation. «Dieu s'est incarné, ce n'est pas pour que nous le désincarnions » (p. 16) d'où la nécessité pour chaque croyant de ne pas dissocier sa situation matérielle de sa situation spirituelle. La foi nous impose la responsabilité d'incarner les valeurs spirituelles dans le domaine matériel et plus généralement dans le monde «auquel nous ne devons pas échapper» (p. 19). En particulier Ellul rejette vigoureusement les doctrines évolutionistes du salut qui croient à une «apparition progressive [du royaume], ascension de l'humanité vers Dieu» (p. 113) C'est donc cette exigence d'incarnation qui pousse Ellul à chercher à construire «une civilisation à hauteur d'homme» (p. 31). Or notre civilisation technicienne n'est pas à la hauteur de cet homme de chair. La croissance de ses moyens économiques, scientifiques et techniques est fondée sur une abstraction qui sacrifie l'homme concret à l'homme idéal: "Ainsi l'homme vivant, concret, l'homme de la rue, est soumis aux moyens qui doivent assurer le bonheur à l'abstraction homme. L'homme des philosophes et des politiciens, qui n'existe pas, est la seule fin de cette prodigieuse aventure qui fait la misère de l'homme de chair et de sang, et le transforme partout en moyen.» (p. 83). Cette attention à la condition incarnée de l'homme conduit Ellul à refuser toute dissociation entre moyens et fins. «Dans l'œuvre de Dieu la fin et les moyens sont identifiés (...) Jésus-Christ dans son incarnation apparaît comme le moyen de Dieu, pour le salut de l'homme, et pour l'établissement du Royaume de Dieu. Mais là où est Jésus-Christ là est aussi ce salut et ce royaume.» (p. 104).

L'incarnation du Verbe en Christ donne donc aux hommes un modèle. L'action des hommes, elle aussi, doit chercher à unir le matériel et le spirituel. De ce que le Verbe s'est fait chair il résulte que toute action doit, aussi bien dans l'esprit et les gestes de celui qui la met en œuvre que dans tous ses effets concrets, incarner le Verbe et ses exigences. Pour qu'une action soit bonne il faut que sa finalité soit incorporée non seulement dans ses effets mais aussi dans l'agent et dans les moyens. Une action efficace mise en œuvre par quelqu'un qui ne sait pas ce qu'il fait, qui est réduit au rang d'instrument irresponsable, ne peut être bonne. «ce qui compte ce ne sont pas nos instruments et nos institutions mais nous-mêmes...» (p. 105). C'est là un principe qu'Ellul répète inlassablement dans nombre de ses livres, à savoir que les moyens doivent être toujours conformes aux fins et qu'on ne peut réaliser des fins justes par des moyens injustes. Le moyen doit être lui aussi une fin et il faut que l'action par laquelle nous mettons ce moyen en œuvre soit elle-même juste dans toutes ses dimensions concrètes. C'est seulement par un processus de désincarnation (qui est au cœur de l'aventure techniciste occidentale) que l'on peut s'imaginer qu'une action peut être justifiée par ses fins. On ne peut moralement justifier le recours à un type d'action qui a des effets négatifs sur

${ }^{22}$ Ellul, Jacques, Présence au monde moderne, Genève, Roulet, 1948, 202 p. 
l'homme (par exemple le travail à la chaîne, la dépersonnalisation administrative ou la violence politique) par la fin qu'il prétend servir. «...nous sommes amenés à dénier le caractère de moyen à toute l'activité purement humaine, à tout ce travail de l'homme qui occupe aujourd'hui notre champ de vision.» (p. 116). Une action qui amoindrit le sujet ou qui dégrade son objet, quelle que soit sa finalité ultime, est mauvaise.

Si donc l'on prend l'incarnation au sérieux il découle que nos actes, tous nos actes et dans tous leurs effets, doivent incarner nos valeurs. Cette exigence n'est pas du tout originale, mais l'originalité d'Ellul consiste à la prendre au sérieux dans toute sa radicalité pour en faire le critère d'une évaluation sans concession de la dépersonnalisation de la vie quotidienne moderne, ce qui le conduit à sa critique de l'Etat et de la technique modernes. Il montre alors comment l'appareillage technique et institutionnel de la société moderne tend à s'autonomiser, ce qui est contradictoire avec exigence d'unité personnelle de la fin et des moyens qui découle de l'incarnation. De ce refus de dissocier fins et moyens Ellul souligne «la conséquence que les chrétiens doivent mettre en pratique, c'est qu'actuellement il s'agit d'être et non pas d'agir.»(p. 119). Ellul insiste en effet à plusieurs reprises sur la primauté de la vie par rapport à l'action. «Dans une civilisation qui ne sait plus ce que c'est que la vie, tout ce que peut faire d'utile un chrétien, c'est précisément de vivre, et la vie comprise dans la foi a une puissance explosive extraordinaire; nous ne le savons plus parce que nous ne croyons plus qu'à l'efficience, et que la vie n'est pas efficiente. Elle peut - et elle seule- provoquer l'éclatement du monde moderne en faisant apparaître aux yeux de tous l'inefficacité des techniques» (p. 124) «Il s'agit donc de retrouver tout ce que signifie la plénitude de la vie personnelle pour un homme planté sur ses pieds, au milieu du monde ...» (p. 125). C'est donc de cet accent mis sur l'incarnation dans le christ comme dans la vie de l'homme que découle pour Ellul la nécessité de soumettre les techniques et les institutions à un jugement qui leur assigne une place dans la vie de l'homme ainsi que des limites.

Ellul insiste beaucoup moins que Charbonneau sur l'importance des dimensions sensibles du rapport charnel au monde naturel. Par contre le même souci d'incarnation sous-tend sa réflexion sur le monde social et en particulier sur les genres de relations de pouvoir et de communication qui s'établissent entre les hommes. Ainsi dans La parole humiliée, Il aborde la question de la désincarnation de l'expérience du sens dans des termes très proches de ceux d'Illich.

Par ailleurs ce souci de l'incarnation conduit Ellul à affirmer la centralité de l'individu, sanctifiée par l'exemple de l'individualité du Christ. Dieu s'est incarné comme individu situé dans l'espace et dans l'histoire: l'individualité est donc une dimension essentielle, sanctifiée, de notre humanité. La vie de jésus donne le modèle accompli de l'incarnation des vérités spirituelles par leur mise en pratique. Cela, seul un individu peut le faire. Et cette mise en pratique des vérités spirituelles doit donc passer par l'action individuelle et doit être de la responsabilité de chaque individu. Il ne suffit pas qu'une technique ou une institution soit impersonnellement efficace, produise automatiquement tel ou tel effet, pour qu'elle soit bonne. Elle doit en outre laisser à chaque individu la possibilité d'être responsable de ses actes pour qu'on puisse dire qu'elle est vraiment bonne. De cette exigence d'incarnation Ellul souligne diverses conséquences : 
Premièrement: Il résulte de l'unité charnelle de l'être humain que les hommes doivent mettre en œuvre cette exigence d'incarnation dans toutes les dimensions de leur vie. Ainsi pour ce qui est des relations de pouvoir il faut être attentif non seulement aux formes politiques mais aussi aux formes non-politiques de domination. Cela suppose que l'on accorde une grande importance aux structures de la vie quotidienne pour appréhender les phénomènes de pouvoir qui s'y déploient.

Deuxièmement: Le souci d'unité entre la pensée et l'action motive la réflexion socio-politique d'Ellul et toute son œuvre met l'accent sur l'exigence d'autonomie personnelle comme condition et réalisation de la liberté. C'est par l'action de chacun que le verbe de Dieu s'incarne dans le monde. D'où l'importance dans la pensée politique d'Ellul de la notion de chacun, «chaque un ${ }^{23}$. Chaque homme est appelé à agir, décider. Très tôt Ellul s'est demandé comment vivre de manière à pouvoir être responsable de ses actes, alors que la société moderne dépersonnalise l'action. Dans les Directives pour un manifeste personnaliste ${ }^{24}$, texte rédigé en 1937 avec Charbonneau, Ellul se révolte contre la dépersonnalisation de l'action et l'anonymat qui résultent du fonctionnement normal des institutions administratives, économiques et techniques de la société moderne. Il s'obstine à évaluer les institutions et les techniques non seulement en termes d'efficacité mais aussi - et surtout - en fonction des conséquences qui en résultent pour la maîtrise de chacun sur ses conditions de vie concrètes; quelle place la civilisation technicienne laisse t'elle au pouvoir de décision de chacun? Pour lui, tout ce qui réduit cette maîtrise dans la vie quotidienne est un mal. «Nous sentions la nécessité de proclamer certaines valeurs et d'incarner certaines forces.» Mais alors que «le problème personnel consistait à se demander si nous pouvions incarner la nécessité que nous sentions en nous», «nulle part il n'était plus question de vivre sa pensée et de penser son action, mais seulement de penser tout court et de gagner sa vie tout court ». Face à une civilisation qui institutionnalise et porte à l'extrême la scission du matériel et du spirituel, Ellul se soucie d'instaurer des conditions de vie qui soient concrètement compatibles avec l'exigence de responsabilité personnelle de l'individu dans tous les domaines de sa vie.

Troisièmement: Les orientations spirituelles et morales doivent se traduire d'abord par le souci du style de vie. L'objectif de changer la vie doit pouvoir se traduire dans toutes les actions de la vie quotidienne. La vie privée est aussi importante que l'action politique.

\section{$*$ \\ $* \quad *$ \\ QUELQUES REMARQUES PHILOSOPHIQUES}

L'esprit du quotidien: Que ce soit chez Charbonneau, Illich ou Ellul, la critique de la dépersonnalisation technicienne est conduite au nom d'une exigence

\footnotetext{
${ }^{23}$ A relier peut-être avec sa conception de l'universalité du salut.

${ }^{24}$ Charbonneau, Bernard et Ellul, Jacques, Directives pour un manifeste personnaliste, Journal intérieur des groupes personnalistes du Sud Ouest, 1935, édition critique annotée par Patrick TroudeChastenet, in Revue d' Histoire des Idées Politiques, n 9, $1^{\text {er }}$ sem. 1999.
} 
d'incarnation qui s'enracine dans la tradition spirituelle judéo-chrétienne. De ce point de vue leurs valeurs ne sont pas du tout originales; ce qui est original par contre c'est leur détermination a juger les aspects les plus quotidiens de la modernité à l'aune de ces valeurs. Sans oublier que chez ces trois auteurs cette critique a été d'abord motivée par une révolte devant les conditions de la vie quotidienne moderne plutôt que par une démarche spéculative, nous allons maintenant proposer quelques jalons qui permettent de baliser a posteriori la logique de cette filiation entre l'expérience judéo-chrétienne de l'existence et la critique du technicisme moderne. Ce faisant on pourra mieux appréhender le fond spirituel commun à ces trois auteurs.

L'incarnation est une notion centrale dans la Bible et semble ne pas avoir d'équivalent dans la tradition philosophique occidentale héritée des grecs ${ }^{25}$. C'est au dix neuvième siècle, alors que l'industrie triomphe partout en Europe que la notion de chair émerge comme un thème important de la philosophie moderne. Elle inspire en particulier la critique phénoménologique et existentielle de la vision scientiste du monde et de l'instrumentalisme techniciste. Contre cette vision du monde qui leur semble avoir tiré son dynamisme de la tradition spéculative héritée des grecs, certains penseurs vont mobiliser une autre tradition: celle de la spiritualité juive et chrétienne. Influencée par l'esprit du Talmud ou celui des Confessions augustiniennes, cette tradition a toujours cherché la vérité plus du côté de l'expérience intime que de l'objectivité impersonnelle et atemporelle du concept. On le voit, si la notion de chair est mobilisée par la philosophie moderne cela tient plus à l'héritage de Jérusalem qu'à celui d'Athènes.

Dans la Bible l'incarnation est présente à la fois comme un donné et comme une exigence à réaliser. L'incarnation est action. Plus précisément action de se faire chair. Dès le récit de la création du monde, l'incarnation est une des dimensions essentielles du mode d'être au monde qui caractérise l'homme. Le mode d'existence de l'homme, décrit comme chair, mixte de matière et d'esprit unis en un corps vivant et sexué, est déclaré être très bon. Rien dans la Bible ne nous invite à dévaloriser le corps et à délivrer l'esprit humain d'une corporéitéobstacle. L'homme est à l'image de Dieu et, réciproquement le Dieu des chrétiens s'est incarné et a assumé totalement la condition d'homme. Dans les évangiles le corps de l'homme, lui aussi appelé à ressusciter, est à l'image du divin et il est aussi saint que son âme. Avec la naissance de Jésus l'incarnation est manifestation (épiphanie) du verbe divin dans le monde (le cantique «Verbum caro factum est» est un chant de Noël). Elle est ensuite union active du spirituel et du temporel dans ce monde à travers les actions de Jésus puis à travers les Actes des apôtres. Jésus guérit aussi bien les corps que les esprits; pour communiquer son message il se sert de symboles et de paraboles autant que d'énoncés généraux. Tout ceci témoigne d'un souci de ne pas dissocier l'esprit du corps et de s'adresser à la globalité spirituelle, physique et sensible de la personne humaine. L'union du spirituel et du matériel, sanctifiée par l'exemple de Jésus, est un bien; de plus cette

${ }^{25}$ Du Timée de Platon aux Ennéades de Plotin, la philosophie grecque parle volontiers de spiritualisation mais jamais d'incarnation, le corps étant considéré comme un mode déficient de l'Etre. Alors que l'idée de l'éternité de l'âme paraissait cohérente aux philosophes de l'Antiquité, celle de la résurrection des corps, prêchée par Saint Paul à Athènes, leur semblait grotesque. 
union doit se réaliser sur le modèle de la relation d'amour dont Jésus donne le modèle.

C'est ainsi que sous l'influence de la tradition judéo-chrétienne certains esprits ont placé cette union de l'esprit et du corps et au centre de leurs préoccupations et de leur vision de l'homme. Et ce sens de l'incarnation a deux dimensions complémentaires: D'un côté il faut que l'Esprit s'incarne dans le monde en mettant ses vérités en pratique; la liberté est action. Mais, inversement, d'un autre côté, si l'homme est réellement et essentiellement chair, indissociablement corps et âme, il résulte de son mode spécifique d'être au monde que tout ce qui touche les dimensions charnelles et sensibles du rapport qu'il entretient avec le monde matériel dans le quel il vit a une grande importance spirituelle et morale. Il en résulte que nos techniques, leurs conséquences sur notre mode de vie ainsi que la qualité des conditions matérielles de l'existence qu'elles engendrent ne peuvent être spirituellement indifférentes. Au contraire, elles doivent faire l'objet de choix moraux. De ce que l'homme est Chair, il doit accorder la plus grande attention à tout ce qui affecte positivement ou négativement l'union du corps et l'esprit. Le maintien, voire la création d'un tel accord est un enjeu essentiel pour l'accomplissement de l'existence humaine et donc pour évaluer la valeur des actions que l'homme entreprend et des techniques qu'il utilise.

Dans cette perspective, prendre au sérieux l'exigence d'incarnation, c'est être attentif au fait que dans une existence humaine le rapport de l'esprit et de la chair est si étroit et essentiel que ce qui advient au corps et aux sens de l'homme affecte forcément l'esprit. L'homme étant chair, sa puissance n'est pas neutre; l'action qu'il exerce sur les choses, les modalités d'utilisation de ses outils techniques, le type d'environnement qu'il façonne, affectent sa sensibilité et retentissent sur la qualité de sa vie intérieure. C'est pourquoi, la liberté humaine ayant désormais le pouvoir de transformer les conditions matérielles de la vie des hommes, il ne suffit pas pour juger nos outils et les manières d'être au monde qu'ils induisent qu'ils soient techniquement et économiquement efficaces, encore faut-il que leur usage ne nous amoindrisse pas dans la vie de notre corps et de notre sensibilité. Il faut constamment se demander en quoi les techniques et les conditions matérielles que nous produisons vont retentir non seulement sur la satisfaction de nos besoins mais aussi sur les diverses dimensions de l'existence humaine prise comme dans sa globalité sensible et intellectuelle, et quels vont être leurs effets sur la qualité du rapport charnel que nous entretenons quotidiennement avec les autres ou avec le monde.

Par exemple, pour ce qui concerne l'expérience de la liberté, l'autonomie de l'individu et sa capacité à décider personnellement dans tous les domaines de la vie quotidienne sont d'une importance au moins aussi grande que les droits politiques dont il jouit. Souffrir de ne pas être maître de ses gestes et d'être soumis à des relations dépersonnalisantes dans sa vie quotidienne, c'est une forme d'amoindrissement de la personne qui est aussi grave, si ce n'est plus, que de ne pas jouir de droits politiques.

Par exemple encore, pour ce qui concerne la satisfaction des besoins, la qualité sensible du rapport aux choses et au monde qui est induit par nos outils et nos techniques est d'une importance aussi grande que la quantité de biens consommables dont nous pouvons disposer. Etre condamné, pour satisfaire ses besoins à accomplir des gestes privés de sens, à vivre dans un environnement laid et quali- 
tativement pauvre, à devoir absorber des nourritures insipides, ce n'est pas indifférent; au contraire, ne plus pouvoir éprouver charnellement dans sa vie quotidienne la diversité et la richesse sensible du monde c'est une des formes du mal.

Vous avez dit Incarnation? Les analyses qui précèdent vont inévitablement susciter l'objection suivante: la plupart des chrétiens sincères n'ont absolument pas tiré de leur foi une attitude critique à l'égard des « dégâts du progrès ». Bien au contraire, depuis le vingtième siècle le catholicisme lui-même n'est-il pas devenu ardemment progressiste?

Il est évident que la manière de comprendre l'incarnation que j'ai caractérisée et montrée à l'œuvre dans la pensée de Charbonneau, Ellul et Illich n'est pas celle de tous les chrétiens. Très différente de cette théologie de l'incarnation, il existe en effet toute une théologie de la spiritualisation qui propose à la liberté humaine une orientation diamétralement opposée, qui consiste à mobiliser toutes les ressources de la technique afin de «poursuivre l'œuvre divine». La poursuite de cette œuvre divine ce serait la transformation de toute la nature par la technique, entreprise qui a pour but ultime la suppression de l'altérité du monde matériel, considéré comme ontologiquement déficient, et la spiritualisation de la matière. Cet objectif favorise chez certains penseurs chrétiens un parti pris résolument technophile et progressiste. Ainsi, à la suite du Dominicain M. D. Chenu. et du jésuite Teilhard de Chardin qui saluait l'explosion de la bombe atomique sur Hiroshima comme la manifestation de l'expansion du divin dans la matière, E. Mounier n'hésite pas à écrire que «la nature s'offre à être recréée par l'homme». Pour ce philosophe, fondateur de la revue Esprit, le sens de la technique réside en ce qu'elle autorise un pouvoir radical de la pensée sur la réalité physique. La recréation de la nature dont il se fait l'avocat n'a pas d'autre sens qu'une hominisation totale de la réalité physique, c'est à dire une domination définitive de la matière par l'esprit. C'est pourquoi Mounier n'a pas de mots assez sévères pour fustiger les précurseurs de la critique écologique qui comme Charbonneau et Ellul ${ }^{26}$ se seraient laissés entraîner par la petite peur du vingtième siècle ${ }^{27}$ et font obstacle à cette glorieuse vocation de l'homme ${ }^{28}$.

Le plus étonnant c'est que cette instrumentalisation du corps a pu être légitimée par des théologiens progressistes au nom d'une certaine compréhension de l'incarnation. J'en donnerai comme exemple le recueil La Technique et l' homme, édité par le Centre Catholique des Intellectuels Francais en juin 1960². A

${ }^{26}$ Il ne nomme pas Charbonneau et Ellul mais il est clair qu'il les vise car il les connaissait bien et s'était déjà opposé à leurs orientations environnementalistes au sein du mouvement Esprit d'avant guerre.

${ }^{27}$ Emmanuel Mounier, «La machine en accusation » in: La petite peur du vingtième siècle, Paris, le Seuil, 1955. On peut considérer ce petit ouvrage comme le bréviaire de l'optimisme technophile qui a imprégné les milieux catholiques progressistes de l'après-guerre.

${ }^{28}$ C'est aussi la raison pour laquelle il se soucie de distinguer l'individu et la personne, principe exclusivement spirituel, distinct de son corps et de ses particularités empiriques physiques sociales et psychologiques (au point qu'il est difficile de la distinguer de l'âme des philosophes et des théologiens idéalistes), alors que Bernard Charbonneau, au contraire, s'y refuse vigoureusement.

${ }_{29}$ M.D. Chenu, «vers une théologie de la technique» in La technique et l' homme, Recherches et Débats du cercle catholique des intellectuels français n 31, Arthème Fayard, Paris, 1960. 
l'époque les mises en garde étaient rares mais elles existaient. Le Meilleur des mondes avait été publié en 1932, La bombe atomique avait été lancée sur Hiroschima en 1945, le livre d'Ellul La technique ou l'enjeu du siècle avait été publié en 1954. Les possibilités de manipulations génétiques du vivant avaient été signalées en France par Jean Rostand; son livre Peut on modifier l'homme? date de 1956. Cybernétique et société de Norbert Wiener avait été traduit en français en 1952 etc. Or on ne trouve dans ce recueil aucune analyse rigoureuse des problèmes concrets posés par la civilisation technicienne. Seuls le travail aliéné et la laideur du monde industriel sont évoqués en passant, mais rien n'est dit sur les problèmes d'environnement, sur la biodiversité, sur la disparition des cultures locales résultant de l'industrialisation agricole, sur les problèmes d'emploi résultant du développement de l'automation etc. bref, rien sur les problèmes d'aujourd'hui qui étaient pourtant déjà signalés à l'époque. Quarante ans plus tard on ne peut qu'être confondu par un tel irénisme.

Quelle conception de l'incarnation a pu favoriser cette cécité, cet optimisme sous-critique? Pour la caractériser je m'en tiendrai ici à la «théologie de la technique» proposée dans ce recueil par le Père M.D. Chenu. Apparemment la conception de l'incarnation qu'il nous propose est assez classique: insistant sur la consubstantialité en l'homme de l'esprit et de la matière, il souligne que «sa perfection ne consiste pas à surmonter une existence-dans-le-monde, comme une conjoncture accidentelle assez pesante, mais à réaliser dans ce monde le plein équilibre ontologique et moral de son être». La dimension technicienne de la vie humaine est donc inséparable de son mode d'être incarné. Jusque là tout va bien mais on relève aussi que cette vocation technicienne de l'homme est décrite dans des termes très intellectualistes et instrumentaux: «l' homme est appelé à pénétrer le monde de son esprit en le rationalisant, comme il rationalise vertueusement son propre corps. Dans ce mouvement la transcendance de l'esprit demeure intacte.» Ce qui conduit le Père Chenu à franchir un pas de plus et à nous expliquer sans sourciller que la vérité humaine et divine sur l'homme «c'est que l'esprit pénètre profondément le domaine du corps, de son propre corps, mais aussi de tout le corps de ce monde, en lui accompli; il en est le démiurge, responsable devant le créateur, à l'œuvre duquel il participe...» Les données de la nature, y compris le corps humain, deviennent ainsi les «matériaux de la liberté», et par son action l'homme «achève la consécration $d u$ monde». Je relève ici que la notion d'esprit est identifiée à celle de raison et que le modèle de l'action de l'esprit sur la matière que nous propose Chenu, c'est celui du démiurge, du Dieu fabricateur des philosophes. Il me semble que cela pose problème: à la limite ce modèle peut être celui du Père créateur, mais il est clair que ce n'est pas du tout celui du Fils fait homme. Le texte de M.D. Chenu finit donc par rabattre le mystère de l'incarnation sur un modèle intellectualiste de la création comme imposition par le Père d'un ordre rationnel au monde de la matière. Or le fils, Verbe incarné, n'agit pas comme un créateur qui informe un matériau. L'image du potier convient au Père mais pas au Fils; l'action de jésus, comme sa parole, s'adresse à des êtres de chair qui attendent un royaume d'amour et de liberté et à qui il est toujours laissé la liberté de répondre ou non à son message. Il est clair que penser l'incarnation non plus à partir du modèle du Fils qui a refusé la tentation de la toute puissance mais à partir du modèle du Père, créateur tout puissant, ne permet pas de penser la question des limites de la technique. 
En effet M. D. Chenu nous explique que la foi ne peut plus se borner à orienter la conduite intérieure de chaque homme, car «lié à la matière, cocréateur $d u$ monde, capable d'inventer ses propres environnements, l'homo technicus fait avancer l' histoire». Soit, mais cette affirmation très générale de la vocation technicienne de l'homme devrait déboucher sur toute une série de questions délicates: comment l'homme doit-il traiter cette création qu'il a désormais le pouvoir de maltraiter et à laquelle il appartient toujours; à quels critères éthiques, à quel rythme, à quels seuils de puissance doit-il subordonner la montée en puissance et l'usage de ses outils techniques; quels environnements techniques, sociaux et naturels sont plus ou moins favorables à son épanouissement social, spirituel et sensible? Manifestement ces questions sont sans intérêt, voire absurdes lorsqu'on envisage l'incarnation comme cocréation du monde et lorsqu'on est convaincu que c'est grâce à la technique humaine qu'il va devenir possible de répondre «à l'attente impatiente de la création, en travail d' enfantement, pour participer à la liberté de la gloire des enfants de Dieu» (rom. 8, cité par Chenu). Dans une telle perspective la question des dégâts du progrès, des limites de la puissance et du choix entre divers modèles techniques de développement n'a plus aucune importance. Tout progrès de la puissance est bon, on les accepte donc tous en bloc.

Ainsi, chez ce théologien la référence à l'incarnation s'accompagne d'une compréhension complètement unilatérale de l'action technicienne, comprise uniquement, à la manière platonicienne, comme action de la raison sur une matière qui lui est extérieure, comme si, de par précisément son statut d'être incarné, la technique ne réagit pas toujours sur l'homme de sorte qu'il est bien imprudent d'affirmer avec le Père Chenu que dans l'action technicienne «la transcendance de l' esprit reste intacte». Mais la fascination pour la puissance d'action de l'esprit sur la matière rend en fait indifférent aux modalités selon laquelle cette puissance retentit sur l'homme de chair. De fait cette notion de chair ne joue aucun rôle dans le texte de Chenu.

On comprend en lisant le Père Chenu que Karl Barth ait pu déplorer l'absence de réflexion de la pensée théologique moderne sur la question de l'incarnation. En lisant ce recueil publié par les intellectuels catholiques (bien entendu à l'époque les protestants disaient exactement la même chose) on comprend aussi que leur progressisme technophile repose sur une compréhension très particulière et unilatérale de la notion d'incarnation. Sous ce terme ils entendent en fait la spiritualisation de la nature, c'est-à-dire, en fin de compte la domination de l'esprit sur le corps, enfin soumis à des exigences spirituelles ${ }^{30}$.

Une certaine vision spiritualiste s'accommode très bien de l'instrumentalisation du corps et de la nature. Mais à une condition: ne pas en reconnaître jusqu'au

${ }^{30}$ Signalons que Charbonneau récuse vigoureusement cette manière de parler de spiritualisation sous couvert d'incarnation: «Incarnation. L'Esprit fait chair, non la chair, la matière, se faisant esprit. L'inverse de l'Evolution, laïque ou theillardienne, où la Matière devient Esprit. Dès l'origine l'atome est gros du progrès vers la noosphère; à ce destin la matière ne saurait échapper. L'Homme peut intervenir, il ne changera rien à l'essentiel. Il n'y a plus de liberté. Par contre si l'esprit se fait chair, matière, réalité, c'est parce qu'il s'en distingue. A l'origine il n'y a pas un mais deux termes: l'Esprit et la chair, aussi différents de nature qu'un homme puisse le concevoir; Bien qu'étroitement confondus et affrontés à cette existence individuelle que nous sommes: l'esprit se fait chair dans la conscience personnelle qui souffre de l'immensité de leur distance.» Trois pas vers la liberté, opuscule inédit, p. 7. 
bout toutes les conséquences. On ne peut qu'être frappé par la myopie des auteurs de ce recueil, qui sont incapables de voir les problèmes d'environnement, de manipulation de l'identité du vivant qui en 1960 étaient déjà énormes. Ils raisonnent comme s'ils étaient à l'époque de Marcelin Berthelot et Ernest Renan, en 1850. En particulier, s'opposant à un spiritualisme contemplatif dépassé, ils ne déduisent de la notion de chair que la nécessité de l'action sur le monde; par contre ils ne savent envisager cette action que comme pouvoir de l'esprit (intellect et volonté) sur la matière, selon un modèle instrumental. Leur spiritualisme reste idéaliste, unilatéral. Ils ne déduisent pas de la notion de chair que l'homme est forcément tout autant objet que sujet des techniques et qu'il faut se préoccuper tout autant des effets que le pouvoir des techniques et des outils a sur sa vie charnelle et son monde vécu que de ceux qu'ils ont sur les choses.

En fin de compte sous couvert d'incarnation cette action de l'esprit sur la matière est pensée comme domination plutôt que comme union: imposition au corps des projet et des modèles de l'esprit. Or, cultiver l'union de l'âme et du corps, sans qu'il y ait de domination de l'une sur l'autre, c'est un objectif très différent.

Vivre une tension entre deux opposés: Il me semble donc que selon que l'on met l'accent sur la spiritualisation de la matière ou bien sur l'incarnation de l'esprit il peut en résulter deux types de rapport à la nature qui seront très différents.

D'un côté on valorise, parfois jusqu'à l'inconditionnalité, tout ce qui permet de réduire l'altérité de ce qui est naturel, et en particulier de la corporéité, considérée comme relevant d'un mode d'être ontologiquement déficient et sans valeur intrinsèque, pur matériau voué à se soumettre à l'ingéniosité humaine et à perdre ainsi progressivement sa nature corporelle qui résiste et fait obstacle à l'esprit ${ }^{31}$.

D'un autre côté l'exigence d'incarnation suppose la reconnaissance de la valeur du mode corporel d'existence comme vis-à-vis nécessaire de l'esprit. Pour le dire en termes religieux, la création n'est pas imparfaite; le monde naturel n'a pas a à être «spiritualisé». Un monde qui ne serait qu'Esprit ne serait ni plus beau ni plus riche, bien au contraire, que ce monde qui est corps (matière) et esprit, et il appartient à l'homme, précisément parce qu'il est chair, d'unir les deux par son action. En termes philosophiques, au lieu de vouloir supprimer l'altérité de la nature corporelle chacun est invité à accepter la difficile tâche qui consiste à mettre en pratique personnellement ses valeurs spirituelles dans un monde naturel (et social) qui est soumis à d'autres logiques et à d'autres forces. Ce n'est qu'à cette condition qu'on donne consistance, durée et, en fin de compte, réalité à ces valeurs. On pourrait ici reprendre la métaphore de Kant au sujet de la colombe qui, éprouvant la résistance de l'air comme un obstacle à vaincre, s'imagine qu'elle volerait plus haut dans le vide, alors que c'est précisément la résistance de l'air qui lui permet de vaincre la pesanteur et de réaliser la beauté du vol. C'est l'existence d'un monde qui lui résiste qui permet à la liberté d'un individu vivant de se déployer.

Comme l'écrivait Charles Péguy dans un passage d'Eve:

${ }^{31}$ On voit ici comment la technophilie peut dériver d'une représentation idéaliste de la liberté. 


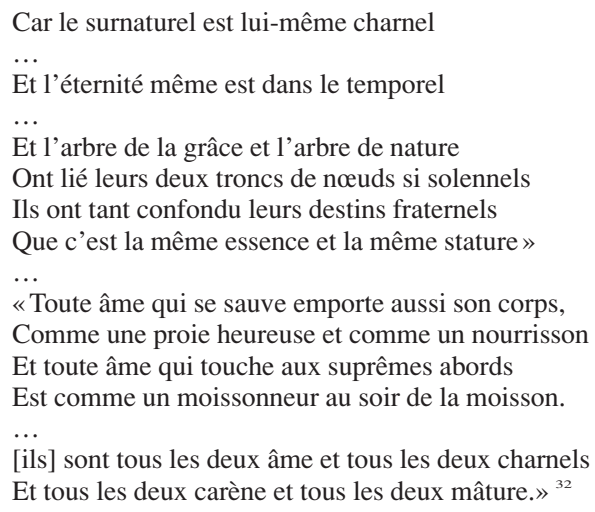

Ici l'accent mis sur l'incarnation incite à maintenir la différence et la tension entre l'ordre de la nature et celui de l'esprit, et non pas à supprimer un des deux termes en l'absorbant dans l'autre. Dans cette perspective le mode d'être au monde de l'homme, qui est celui d'un esprit conscient uni à un corps propre singulier et périssable ${ }^{33}$ n'est pas ontologiquement déficient: dès que les deux termes esprit et corps sont données ensemble, chaque individu humain est suffisamment équipé pour incarner, c'est-à-dire mettre en pratique, ses valeurs spirituelles dans un monde qui ne les connaît pas. Et chaque fois qu'il y parvient de manière réellement personnelle, même si c'est dans le plus humble des gestes, même si c'est dans l'obscurité la plus totale, alors le miracle de la liberté s'accomplit: on peut être plus intelligent, plus puissant, on peut vivre plus longtemps, on ne peut être plus humain. Cela, seul un individu concret, soumis par son corps aux déterminismes de la nature, et par son esprit à ceux de la société ou de son propre inconscient, mais capable aussi de mobiliser la force de son esprit pour leur résister, peut le réaliser.

Certes, comme le reconnaissait Emerson, rien n'est plus rare qu'un acte authentiquement personnel, c'est-à-dire accompli par un individu qui s'efforce de dépasser les déterminations naturelles, sociales et psychologiques qui pèsent sur lui et en lui pour traduire ses valeurs dans la réalité. Peut être qu'aucun de nos actes ne réalise totalement cette exigence; il n'en reste pas moins que tout acte qui réalise un tant soit peu cette incarnation est bon et beau et c'est bien cela qui mériterait d'être sauvé pour l'éternité. A l'opposé, quelque soit le niveau de maîtrise collective de la nature auquel nous parviendrons, nous savons d'instinct qu'une existence dont les actes auraient perdu tout caractère personnel n'aurait plus rien d'humain ${ }^{34}$.

${ }^{32}$ Charles Péguy, «Eve» in Euvres poétiques complètes, pp. 1041-1042. Bibliothèque de la Pléiade, Gallimard, Paris, 1957. Je remercie Guy Roustang de m'avoir signalé ces vers.

${ }_{33}$ Situation qui échappe au pouvoir explicatif de nos concepts et que la pensée religieuse peut déjà, et à juste titre, considérer comme miraculeuse.

${ }^{34}$ C'est bien de ce risque qu'Aldous Huxley cherchait à nous avertir dans Le meilleur des mondes (1931). 
Technique et liberté: L'idée d'imposer des limites à la technique répugne à l'esprit moderne. Cela se comprend fort bien car le progressisme techniciste s'enracine dans une certaine expérience spontanée de la liberté d'agir. Etre libre c'est ne pas subir. Etre plus libre c'est devenir moins dépendant des forces qui s'exercent sur moi et plus généralement des cadres spatio-temporels de l'existence, c'est donc devenir plus fort, plus puissant. Toute augmentation de la puissance est donc considérée comme un progrès de la liberté. En effet augmenter la puissance d'action d'un sujet c'est en fin de compte donner à l'entendement la possibilité d'examiner un éventail plus grand de possibles, c'est donner à la volonté un plus grand nombre d'occasions de choisir ses fins. La puissance est bonne car elle augmente la liberté en multipliant les possibles. Dans une telle perspective il n'y a aucune raison de limiter la puissance technique de l'homme qui élargit sans cesse l'éventail des possibles. Non seulement elle nous permet de choisir et non plus de subir notre relation au monde naturel mais en fin de compte l'augmentation de cette puissance d'action de l'homme sur le monde lui permet un dépassement de son mode «naturel» d'existence et de ses limites physiques et psychiques. Elle autorise un dépassement des limites ontologiques liées au mode d'être naturel de l'homme. Cette expérience du lien entre puissance et liberté favorise une certaine dissociation du corps et de l'esprit. Certes la puissance d'action est d'abord éprouvée comme étant celle du corps, mais la liberté est celle de l'esprit qui analyse, qui délibère, qui choisit et qui veut. C'est pourquoi le complément de la liberté d'agir c'est la découverte de la liberté intérieure et du fait que celle-ci ne cesse de buter sur la résistance que lui oppose le monde des corps et des choses.

Ainsi sur la base de cette scission on voit de développer deux conceptions différentes de la liberté. L'une suppose une distinction stricte entre le sujet qui examine, qui choisit et qui veut et le monde naturel sur lequel il agit. Le corps est alors logiquement représenté comme l'instrument de l'esprit pour agir sur le monde et surmonter les entraves liées à la corporéité de l'existence. La liberté est recherchée dans la déliaison. Par ailleurs ce dualisme peut parfois entretenir une fascination pour le «post humain» qui n'est que la conséquence ultime de l'instrumentalisation du corps par l'esprit. Il n'y a plus aucune raison d'imposer des limites à la puissance d'agir de l'homme, en particulier sur lui-même. La puissance est liberté.

Si l'on refuse cette scission Corps/Esprit et que l'on considère que l'homme de chair est une liberté qui fait aussi partie de la nature, alors on peut poser autrement la question des limites de la puissance technique. De cette condition de chair on peut tirer des indications sur la manière dont l'homme peut orienter sa liberté et l'usage de sa puissance technique.

Rappelons au préalable que l'on peut fonder la nécessité d'imposer des limites à la puissance technique de l'homme sur la nature (hors de lui et en lui) de plusieurs manières:

- prudentiellement: (l'écologie scientifique) L'homme est nature au sein de la nature et c'est en tant que corps vivant dépendant des équilibres de la biosphère qu'il est obligé de mettre des limites aux atteintes qu'il peut infliger au milieu.

- objectivement: on peut invoquer la considération de la valeur intrinsèque des êtres naturels et moraux sur lesquels l'homme peut agir et de leur mode d'exis- 
tence qu'il ne faut pas altérer. Il y a des réalités qui en elles mêmes méritent qu'on ne les appauvrisse pas, qu'on ne les altère pas, qu'on ne les détruise pas: la nature humaine, la richesse, diversité de la création, la splendeur du monde voulu par Dieu etc.

- relativement à la condition humaine: Une troisième approche est possible: c'est celle qu'ont défrichée des penseurs comme Charbonneau Ellul et Illich qui se préoccupent de ce que l'augmentation de sa propre puissance fait à l'homme de chair. Toute augmentation de la puissance induit un certain type de rapport au monde, à soi et aux autres qui peut avoir des effets négatifs pour l'homme et pour sa liberté. On peut bien affirmer la liberté totale de l'homme, il n'en reste pas moins que sa liberté ne peut se déployer que sous certaines conditions. Il a besoin d'un vis-à-vis non humain: d'une nature; et les divers types de rapport qu'il peut établir avec la nature ne sont pas équivalents pour lui. Par exemple certaines modalités de l'action de l'homme sur le monde peuvent conduire à un appauvrissement de sa vie sensible qui n'est pas désirable. Il peut aussi en résulter une réduction de ses marges d'autonomie personnelle et, en fin de compte une remise en cause de l'éthicité de l'homme. La liberté d'un être de chair ne peut se réduire à l'augmentation de la puissance, l'accomplissement de la liberté humaine ne peut résider dans la déliaison. C'est pourquoi une certaine critique philosophique du technicisme est faite au nom de l'unité charnelle des deux opposés que sont l'esprit et le corps de l'homme. Les œuvres de philosophes comme Michel Henry ou Hans Jonas témoignent de la fécondité de cette approche.

Politiquement on ne peut éviter de poser tôt ou tard la question des limites des technosciences et de leur fondement: la relation entre puissance et liberté est trop ambiguë et contradictoire pour qu'on puisse différer indéfiniment certains choix. En effet, à partir du moment où la liberté humaine acquiert la puissance d'agir sur les choses, sur le corps vivant, sur l'organisation sociale, en les objectivant, alors se pose la question de choisir entre les divers mondes possibles que notre puissance opératoire peut engendrer. Il me semble, à la suite de Charbonneau, d'Ellul et d'Illich, qu'il est possible de mobiliser la notion d'incarnation- ou celle de chair- pour guider nos choix sans impliquer de référence à une norme transcendante autre que la liberté.

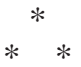

\section{CONCLUSION \\ DE L'INCARNATION À UNE PENSÉE DES LIMITES}

Parce que l'homme est un être de chair, son rapport au monde n'est pas seulement intellectuel ou opératoire mais aussi sensible et symbolique, et ces diverses dimensions retentissent l'une sur l'autre. S'il y a des dimensions matérielles et intellectuelles de la liberté, il y a aussi nécessairement des dimensions sensibles et des dimensions symboliques. La sensibilité est une manière d'entrer en relation avec le monde tout aussi importante que l'action ou la connaissance. Grâce à ses savoir-faire techniques l'homme a la possibilité de réagir et de modifier son 
environnement matériel et de se créer un environnement propre. Mais il ne suffit pas que le nouvel agencement de son environnement favorise une action efficace et permette à l'homme d'exercer sa puissance sur les choses par exemple en multipliant la quantité de bien consommables. Parce qu'elles engagent la totalité de la personne, et qu'elles participent à l'édification d'un monde, les conditions matérielles et spatiales dans lesquelles vit l'homme ne sont pas neutres, au contraire elles sont de la plus haute importance pour la liberté et pour la vie spirituelle de l'homme, de sorte qu'elles ne sauraient être appréciées correctement uniquement en termes de puissance et d'efficacité sur les choses; elles doivent satisfaire tous les registres de sa sensibilité. De l'attention à la globalité de la personne incarnée il résulte que les dimensions sensibles, esthétiques de notre rapport au monde ne peuvent être disqualifiées comme relevant de préférences subjectives inessentielles. Certes lorsqu'on appréhende l'homme comme pure subjectivité, susceptible d'une infinité de préférences, la question de la valeur de son rapport sensible au monde reste indécidable et frivole. Cependant dès lors que l'on reconnaît l'homme d'abord comme un être de chair on peut disposer d'un fil conducteur pour examiner si l'environnement technique ou institutionnel qu'il produit est bien à sa mesure. On peut illustrer une telle démarche par une brève réflexion sur l'impact non plus spatial mais temporel du développement technoscientifique:

Le rapport au monde d'un être de chair est - entre autres - symbolique. Esprit incarné, l'homme a besoin non seulement de techniques, d'une économie, de savoirs exacts et efficaces mais aussi il a besoin de sens. Distincte de l'expérience intellectuelle de la vérité, l'expérience du sens est à la fois spirituelle et physique, elle engage la totalité de la personne qui l'éprouve et qu'elle met en mouvement. C'est pour cela que dès son origine Homo faber est créateur non seulement d'outils mais aussi d'images: son rapport au monde est médiatisé par des symboles qui donnent sens au monde, aux situations qu'il vit et à ses actions. En particulier l'usage des outils, et la puissance qui résulte des opérations techniques ont toujours été intégrées au monde humain et humanisées par une production symbolique. L'être de chair a besoin de vivre au sein d'une civilisation qui inscrit la puissance des techniques dans un ordre symbolique plus vaste, qui organise les relations que les hommes ont entre eux et avec le monde. En retour ce monde symbolique, qui varie selon les civilisations, fournit à chaque individu le sol à partir duquel il peut se construire comme personne. Les transformations des techniques, l'accès à de nouveaux degrés ou même à de nouvelles formes de puissance ont toujours retenti sur l'univers symbolique des hommes, selon un processus d'interaction. D'un côté les innovations techniques appellent par exemple de nouvelles manières de vivre ensemble; d'un autre côté les constructions symboliques imposent en retour des limites à la puissance des nouvelles techniques, limites au delà des quelles leur usage ne peut être ni bon, ni beau ni juste. L'homme de chair a donc besoins de vivre dans une civilisation.

Or, toujours parce que l'homme est chair, engagé corps et âme dans un monde, la production symbolique, sans laquelle une civilisation ne peut s'édifier, a une temporalité spécifique. Elle s'élabore très lentement. L'inconscient y a une large part. Pour que la culture symbolique soit efficace, il faut que chacun en intériorise les modèles de relation à autrui et au monde. Ces modèles s'élaborent et évoluent lentement, sur plusieurs générations. Ils se sédimentent et se transmettent sous la forme d'une tradition qui fournit le terreau indispensable dans lequel peuvent 
s'enraciner les individus pour se construire comme sujets. Si ce capital symbolique ne se transmet pas ou se transmet mal, les individus auront du mal à se construire et la vie collective risque de se désorganiser. Par ailleurs les schémas symboliques reçus au cours de la petite enfance tiennent littéralement à la peau des hommes. Les remettre en cause c'est les écorcher, remettre en cause leur identité et susciter toutes sorte de réactions qui peuvent être très violentes. On ne se défait pas par décret d'un tel héritage, comme l'a amplement prouvé l'échec des révolutions modernes qui ont prétendu créer un «homme nouveau». Or, dans la société moderne, la temporalité sourde de la création symbolique reste beaucoup plus lente que la temporalité du changement technique qui ne cesse de s'accélérer. Le temps de l'innovation technique et celui de créativité culturelle sont devenus complètement hétérogènes, de sorte que l'innovation technique est désormais bien trop rapide pour pouvoir être accompagnée par une production symbolique susceptible d'intégrer les nouveaux pouvoirs dans un nouvel ordre cohérent disons une nouvelle civilisation. Par contre, révolutionnant toujours plus vite la base matérielle de la vie collective, l'innovation technique disqualifie les univers symboliques, fait obstacle à leur transmission et, en fin de compte, détruit les civilisations existantes. Le rythme de l'innovation est tel que les efforts pour combler le vide sont déjà dépassés avant même d'avoir pu aboutir. La société en développement rapide est donc en proie à de nombreuses formes de désorganisation non seulement économiques mais aussi psychologiques, culturelles et sociales, qu'il s'agisse de la construction individuelle des personnes, des rapports que les hommes ont entre eux ou avec la nature. Il suffit pour s'en rendre compte de regarder le journal télévisé ou de jeter un œil sur les gros titres de la presse quotidienne.

Parce que l'homme est un être de chair, la construction de sujets humains équilibrés, institués comme sujets, l'instauration de modes de coexistence (le lien social) préservant un minimum d'autonomie et de solidarité, et enfin l'établissement d'un compromis satisfaisant entre la puissance technique et la diversité et les équilibres naturels, tout cela requiert une temporalité lente; autant d'exigences difficilement compatibles avec le «déferlement technologique ${ }^{35}$ contemporain. On peut donc penser que la prise en considération attentive du statut de chair qui est celui de l'homme pourrait fonder la nécessité d'imposer un rythme beaucoup plus lent au changement technique. Ainsi, à ceux qui souhaitent entretenir ce déferlement technologique, on pourrait objecter que leur politique se fonde sur une conception désincarnée de l'existence et de la liberté humaines et sur un refus de prendre en compte le mode d'être au monde qui caractérisera l'homme aussi longtemps qu'il sera un esprit individuel incarné dans un corps mortel; refus qui peut favoriser bien des situations catastrophiques et déshumanisantes.

Ainsi la prise en considération de la dimension charnelle de l'existence humaine peut fonder le souci d'imposer une autre temporalité au changement technique. Il ne s'agit que d'un exemple particulier d'une démarche que les œuvres de Charbonneau, Illich et Ellul nous invitent à généraliser en l'appliquant à toutes les dimensions de la vie sociale. Alors que la question des limites de l'opérativité technoscientifique se pose de manière de plus en plus aigue, ils nous

\footnotetext{
${ }^{35}$ Pour reprendre la belle formule de Michel Tibon-Cornillot.
} 
invitent à nous appuyer sur l'exigence d'incarnation de l'esprit pour orienter notre liberté et pour faire en sorte que le dynamisme technicien, qui est une des manifestations de cette liberté, ne se retourne pas contre elle. Face aux risques de dépersonnalisation de l'existence qui résultent de la fascination pour une liberté désincarnée, s'adossant à la tradition spirituelle judéochrétienne, ces trois auteurs invitent l' homme de chair à cultiver ce que Nietzsche appelait «le sens de la terre $»^{36}$.

${ }^{36}$ F. Nietzsche, Ainsi parlait Zarathoustra, prologue. 